\title{
Image de force, perception de faiblesse: La clémence d'Henri IV
}

\author{
MICHEL DE WAELE
}

Résumé: Parmi les nombreux éléments qui composent la légende d'Henri IV, la clémence qu'il manifesta envers ses ennemis occupe une place de choix. Sans elle, affirment de nombreuses personnes, le premier Bourbon n'aurait jamais pu s'asseoir sur le trône de France. Tous ne partageaient pas cependant cet enthousiasme à la fin des guerres de religion. Selon plusieurs partisans du roi, cette clémence excessive, loin de montrer la puissance du Béarnais, témoignait plutôt de sa faiblesse.

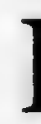

Le 6 juin 1598, menée par le premier président Achille de Harlay, une délégation de parlementaires parisiens se présente au Louvres pour féliciter Henri IV de sa victoire sur le duc de Mercoeur qui tenait jusque là la Bretagne au nom de la Ligue, le parti des catholiques extrémistes lors des guerres de religion. La reddition du duc faisait en sorte que toute la France reconnaissait maintenant le premier Bourbon pour son souverain légitime; l'ensemble du royaume était pacifié. Harlay, dans le compliment qu'il adresse au roi à cette occasion, résume les moyens utilisés par ce dernier pour mener à bien la pacification de son État:

La réputation de votre vertu guerrière formidable à ces mutins ne les a pas seule dompté, l'espérance ou plutôt la certitude de jouir des effets de votre clémence épandue en tous endroits de ce royaume vous ont donné cette dernière victoire si honorable qu'aucuns n'en reçoient plus de commodités que les vaincus outre ce qu'ils ont évité l'expiation exemplaire de leur perfidie à laquelle toute la France jugeait que par ordonnance divine ils étaient réservés. ${ }^{1}$

Dès le début de son règne, Henri IV tenta de gagner l'appui de ses sujets rebelles en leur promettant d'oublier leurs fautes passées pour peu qu'ils acceptent de rejoindre son parti. Le 9 décembre 1589, le parlement de Tours, 
formé de magistrats royalistes qui avaient fui Paris, tombée aux mains des rebelles catholiques, enregistre des lettres patentes du roi qui officialisaient cet engagement. ${ }^{2}$ Bien que certains de ses partisans la trouvent fort excessive - on peut détecter une certaine réserve, voire une rancoeur dans le discours de Harlay cité plus haut lorsqu'il dit que ce sont les vaincus qui tirent le plus de bénéfices de la pacification du royaume - la magnanimité du nouveau roi devint rapidement un des thèmes centraux de la propagande royale.

Ceux qui montraient un certain scepticisme quant aux résultats éventuels de cette politique considéraient la clémence comme une arme à double tranchant. À leur avis, bien dosée, elle permettait éventuellement au souverain et à son peuple de vivre en sécurité et dans la paix. Démesurée par contre, elle risquait d'ouvrir la porte aux abus, les malfaisants se sachant assurés de leur impunité. Si tous célébraient la clémence comme vertu royale, certaines interrogations subsistaient donc quant à son application pratique. En promettant l'oubli de leurs fautes à ses ennemis avant même d'entreprendre la lutte contre eux, Henri IV ne leur transmettait-il pas le message qu'il était trop faible pour les combattre? Ne devait-il pas attendre sa victoire finale avant de leur pardonner leurs fautes? Associée généralement à l'idée du vainqueur, et donc à une image de force, la clémence pouvait ainsi être perçue comme une manifestation de faiblesse. Ce double aspect, nous le retrouvons dans les débats qui opposent partisans et adversaires de la magnanimité royale au cours des premières années du règne d'Henri IV.

Les Français savaient fort peu de choses de cet Henri de Navarre qui devenait soudainement leur roi suite à l'assassinat d'Henri III. Élevé loin de la cour, en lutte presque continuelle contre le roi de France depuis 1576, le Béarnais n'était pour ainsi dire connu de ses sujets que par le biais de pamphlets écrits sur lui par ses ennemis. ${ }^{3}$ Premier représentant d'une nouvelle dynastie, Henri IV devait montrer à ses sujets qu'il était vraiment digne de monter sur le trône de saint Louis. Il devait leur prouver qu'il possédait les qualités requises pour replacer le royaume au niveau de sa plus grande splendeur. Cet objectif devait être atteint le plus rapidement possible, de nombreuses personnes contestant ses droits à la couronne. Non seulement le Béarnais devait-il conquérir militairement son royaume - il n'en contrôlait qu'un sixième lors de son accession au trône -, mais il devait également s'emparer au plus tôt de l'âme de ses sujets.

Ce double défi militaire et moral n'était pas facile à relever. Les exploits guerriers d'Henri de Navarre ne garantissaient pas pour autant sa reconnaissance en tant que souverain par l'ensemble des Français. Il se devait également d'agir en roi pour prouver que la France se trouvait réellement sur la 
voie d'un renouvellement devenu nécessaire suite au déclin du royaume survenu sous les derniers Valois. Il ne pouvait compter pour ce faire sur les éléments symboliques traditionnels qui assuraient au roi de France son caractère mystique, vaguement surnaturel et assurément unique. Le sacre, si important puisque durant cette cérémonie le monarque était oint du saint chrême, la guérison des écrouelles conféraient cette odeur de sainteté aux souverains des fleurs de lys.

Mais la foi protestante d'Henri IV le coupait de ces symboles. Il ne touchera les écrouelles que le 10 avril 1594, jour de Pâques. Il avait été sacré à Chartres le 27 février précédent après s'être converti au catholicisme en juillet 1593. Il lui fallait donc adopter au plus vite un comportement associé à l'image royale. Et quelle meilleure façon de montrer aux yeux de tous que l'on est véritablement un roi que d'exercer un privilège réservé strictement aux seuls souverains: le droit de grâce? ${ }^{4}$

Dès le début de son règne Henri IV présente donc un visage bienveillant à ses sujets, leur promettant d'oublier leurs fautes passées en échange de leur ralliemént à sa cause. Dès 1590 , ses propagandistes feront une large part à cette vertu dans leurs discours. Selon l'avocat Antoine Arnauld, la clémence du roi est "ouverte et très assurée: elle surpasse toutes nos fautes, elle est immense"; "Tous craindront, mais peu seront punis" renchérit un autre royaliste cette même année. ${ }^{5}$ Cette clémence servira à asseoir la légitimité monarchique du Béarnais mais aussi à le rapprocher de ses sujets, à les attacher à sa personne. Dans une lettre adressée au chancelier Pomponne de Bellièvre le 18 avril 1603, l'ancien ligueur Louis Dorléans fait d'ailleurs le constat suivant: “Que si le roi n'eut pardonné à qui eut-il commandé car toute la France était contre lui et à présent elle est glorieusement à lui." 6

Le pardon est un événement mettant en présence deux individus: la personne qui requiert la grâce et celle qui détient le pouvoir de la lui accorder ou de la lui refuser. ${ }^{7}$ Personne n'a droit automatiquement à la clémence; l'offrir ne constitue pas une obligation pour le roi. Elle représente quelque chose de définitif impliquant une déviation consciente de certaines règles publiques par l'autorité possèdant le pouvoir suprême sur ces dites règles. Le pardon devient un triomphe complet du souverain sur son sujet. Impliquant généralement la reconnaissance d'une culpabilité, il exige le repentir du demandeur ou à tout le moins son passage par un certain "rituel d'humiliation." En contrepartie de la rémission de sa peine ou de l'oubli de ses fautes, le condamné ou le suspect se donne complètement au roi. La gratitude éprouvée par le sujet qui, dès lors, a contracté une dette morale et/ou politique envers son souverain doit garantir sa fidélité à celui-ci. ${ }^{8}$ 
Ce contact personnel qui s'établira ainsi entre Henri IV et ses sujets fera sa force. Les édits de pacification de ses prédécesseurs Charles IX et Henri III s'appliquaient à l'ensemble du royaume. L'amplitude de leur juridiction leur donnait un aspect impersonnel évident à ces mesures. La démarche qu'empruntera le Béarnais sera différente. Bien sûr, la juridiction de l'Édit de Nantes promulgué en 1598 couvrait la France entière. Mais, auparavant, Henri IV s'était attaché à pacifier son royaume, ville après ville, province après province. Dès qu'une municipalité ou une région se mettait sous sa tutelle, le roi proclamait une ordonnance de paix qui ne concernait que celle-ci, et dans laquelle on retrouvait fréquemment des clauses ne s'appliquant de façon spécifique qu'à cette ville ou à cette région. ${ }^{9}$

Les différents édits d'Henri IV n'auraient pu suffire à pacifier le royaume. Sa magnanimité s'inscrivait dans un processus plus large de pacification nationale qui se terminera avec la promulgation de l'Édit de Nantes et qui sera marqué entre autres par sa conversion au catholicisme et par la déclaration de la guerre contre l'Espagne en 1595. Au plan des relations avec les individus, Henri IV devait également inciter les nobles à réintégrer le parti royal. Le roi leur offrira pour ce faire des avantages monétaires et sociaux immenses. Les coûts excessifs engendrés par l'achat des fidélités des chefs ligueurs feront sourciller certains proches conseillers du roi. Leurs reproches porteront tout autant sur le principe de ce que l'on pourrait appeler la "rémunération de la révolte" que sur des privilèges particuliers consentis à ces occasions. La jalousie n'était pas absente chez certains collaborateurs d'Henri IV, la part du butin qu'ils escomptaient se retrouvant parfois dans les goussets de leurs anciens adversaires.

Ces critiques s'ajoutent aux réactions mitigées provoquées chez certains par la clémence du roi. L'indulgence du Béarnais à l'égard de ses anciens ennemis déconcerte en effet bon nombre de ses partisans: "ses ennemis trop volontiers, et nous à regret souvent confessons, que c'est le plus doux, le plus pardonnant, et le plus oublieux d'injures qui fut onc." 10 Influencés par la philosophie stoïcienne, certains royalistes percevaient la justice comme la récompense des bons et la punition des méchants. ${ }^{11}$ Pour eux, la magnanimité excessive d'un monarque s'apparentait à l'injustice. La clémence, croyaientils, devait n'être exercée que par un roi vainqueur, un souverain en position de force. La "Harangue de Monsieur d'Aubray," tirée de la Satyre Ménipée publiée en 1593, exprime clairement ce point de vue: "Concluons donc que notre roi devrait réserver à user de sa clémence, quand il nous aura tous en sa puissance. C'est inclémence voire cruauté, dit Cicéron, de pardonner à ceux qui méritent mourir." La Satyre Ménipée, écrit représentatif de la pensée du 
groupe des Politiques, ces catholiques opposés à l'extrémisme de la Ligue et qui étaient prêts à reconnaître Henri IV pour peu qu'il se convertisse au catholicisme, se montre très sévère au sujet de la clémence manifestée par le Béarnais: la magnanimité d'un prince peut être attribuée "à couardise et timidité, plutôt qu'à vaillance et générosité"; "La malice des rebelles s'opiniatre et s'endurcit par la douceur dont on use envers eux."12

Plusieurs raisons peuvent expliquer les réserves de certains partisans d'Henri IV face à sa magnanimité. Il existe tout d'abord des motivations politiques. Charles IX et Henri III avaient tenté vainement de mettre un terme aux guerres de religion en usant de douceur. Le Béarnais, en empruntant cette même voie, ne risquait-il pas lui-même de subir un échec cuisant? Cette option était considérée d' autant plus dangereuse que, pour certains, elle avait conduit à l'assassinat du dernier Valois. Henri III aurait en effet ultimement payé de sa vie sa bienveillance à l'égard de ses ennemis. ${ }^{13}$ César lui-même, considéré à cette époque comme le plus bel exemple de clémence royale, n'était-il pas mort de la main d'individus à qui il avait pardonné leur implication dans un complot contre sa personne ${ }^{14}$

La voie choisie par Henri IV pour pacifier son royaume pouvait en effet s'avérer dangereuse. En oubliant les fautes de ses sujets, le Béarnais ne pouvait s'assurer du degré du repentir de ceux-ci. En fait, il n'en avait aucune idée. ${ }^{15}$ Les Ligueurs allaient-ils profiter de cette pause dans les hostilités offerte par le roi pour refaire leurs forces? Avaient-ils réellement accepté la victoire finale du parti royaliste? De nombreux partisans d'Henri IV craignaient une résurgence de la Ligue pour peu que les affaires du royaume tournent mal. Malgré la fin des guerres civiles, les Français continuent à souffrir de la famine, de la cherté des produits et des affronts des gens de guerre. En décembre 1596, le conseiller au parlement de Paris, Jacques Gillot, signale dans une lettre au président Jacques Auguste de Thou que des individus travaillaient activement en vue d'une résurrection de la Ligue. ${ }^{16}$ Arrivant en Provence en 1599, Guillaume Du Vair affirme y avoir trouvé beaucoup de pauvreté et des divisions de toutes sortes parmi la population. Il craint les conséquences de la promulgation de l'Édit de Nantes sur des esprits déja échauffés. ${ }^{17}$

Pour certains partisans d'Henri IV, il semblait nécessaire de faire un exemple en punissant au moins les chefs de la rébellion. Selon le pamphlétaire royaliste Daniel Drouin qui écrit en 1592, un État ne peut se maintenir si son gouverneur ne fait pas preuve occasionnellement d'un minimum de sévérité. Il poursuit en affirmant que "Le roi par une trop grande facilité et douceur ne fait non moins de dommage que par tyrannie". ${ }^{18}$ L'auteur fonde son argu- 
mentation sur les précédents établis par les rois de France. Bien que, selon Drouin, ils se fussent toujours montrés moins sévères que les monarques des autres nations envers leurs sujets rebelles, les souverains français n'avaient épargné ni cordes, ni potences, ni roues et autres supplices pour punir les séditieux selon leurs crimes. Chilpéric IV, Louis le Gros, Philippe le Bel, Charles VI auraient tous pris soin d'exécuter les chefs des révoltés qui s'étaient élevés contre eux. ${ }^{19}$

Faire preuve d'une telle justice est, selon plusieurs auteurs de l'époque, essentiel pour un prince. Pardonner à tout venant, c'est faire preuve de lâcheté affirme le président L'Allouette. Michel Hurault, parlant d'Henri III en 1588, "le premier roi lequel on a pu hardiment et sans crainte offenser," soutenait qu'un excès de clémence et de douceur pouvait être associé à de la nonchalance. ${ }^{20}$ En refusant d'examiner les fautes de certains de ses sujets, en les oubliant, Henri IV ne faisait-il pas la preuve que le sens de l'équité lui manquait? Ne laissait-il pas paraître qu'il était peu ou pas intéressé à redorer le blason de la justice? Le redressement de la France que l'on attendait sous sa férule impliquait aussi une remise à l'honneur du processus judiciaire. Plusieurs Français considéraient que celui-ci avait été foulé aux pieds lors des guerres de religion, que plus personne ne respectait l'idéal de justice. ${ }^{21} \mathrm{Le}$ rétablissement de la monarchie, la prise en main des affaires par un roi légitime, gonflent les espoirs de certains partisans d'Henri IV: "La force des lois reverdira, l'autorité des cours reluira, la fermeté des jugements s'établira." 22 En offrant sa clémence avant même de s'être penché sur les fautes de ses sujets, Henri IV pouvait donner l'impression de faire peu de cas du processus judiciaire et du devoir du prince d'assurer la justice à ses sujets. Ce grief était d'autant plus grave que de nombreux auteurs considéraient alors que le premier devoir d'un souverain était justement d'assurer la justice à ses sujets. $^{23}$

Se basant sur cette affirmation, des partisans d'Henri IV s'opposeront à ce que des officiers ayant choisi le parti de la Ligue puissent réintégrer leur charge sans coup férir. François de Clary livre ainsi un plaidoyer passionné en 1591 dans lequel il s'indigne de voir la grâce royale s'étendre aux officiers qui avaient refusé de suivre le parti du roi légitime. ${ }^{24} \mathrm{En}$ fait, si tous célèbrent la clémence royale, les principales interrogations à son sujet concernent le degré de sa mise en application. Un homme naturellement turbulent ne saurait être changé par la douceur dont on use envers lui, selon Guillaume Joly. Pierre Poisson de la Bodinière croyait quant à lui qu'il fallait éviter des châtiments trop sévères, ceux-ci produisant généralement un désir de vengeance chez le 
condamné. Toutefois, il reconnaissait que les sujets devaient au moins craindre modérément leur prince. ${ }^{25}$

Les discussions sur ce sujet, il faut le signaler, n'étaient pas nouvelles. Déja, lors des États-Généraux de 1560, le clergé et le Tiers État avaient critiqué l'indulgence des souverains envers les criminels. On s'interrogeait également sur le statut des personnes qui voyaient leurs crimes pardonnés par le monarque. Ce processus était-il équitable pour toutes les couches de la société ou le roi ne viciait-il pas le processus judiciaire pour protéger certains privilégiés? ${ }^{26}$

L'étude de la clémence manifestée par Henri IV à la fin des guerres de religion permet de soulever certaines questions relatives à la vie politique et sociale de cette époque. L'attitude du roi qui ne condamne à l'exil que quelques membres du Tiers État - curés, marchands, artisans ou petits procureurs - et qui laisse le parlement exécuter quelques individus de basse condition convaincus d'avoir trempé dans l'assassinat du président Brisson et des conseillers Larcher et Tardif illustre-t-elle d'une certaine façon les forces sociales à l'oeuvre en cette fin du $\mathrm{XVI}^{\mathrm{e}}$ siècle? Il semble que l'on condamnait alors plus facilement un roturier qu'un noble. Si Henri IV a toujours prétendu que le bien-être du peuple français était pour lui primordial, ce sentiment ne l'empêche pas d'accorder plus de valeur à la vie d'un membre de la noblesse qu'à celle d'un individu issu du Tiers État.

L'"oubliance" générale décrétée par Henri IV peut nous fournir également quelques indications sur les "droits et libertés" relatifs à la guerre à cette époque. Dans ses édits de pacification, le roi n'établit en effet aucune discrimination dans les crimes pardonnés. Les gestes les plus sordides, ceux commis par traîtrise ou perfidie ne seront pas retenus contre leurs auteurs. Ceci allait à l'encontre des coutumes criminelles du temps qui reconnaissaient seulement quelques actes comme étant rémissibles. En 1576, le juriste Jean Papon retenait onze circonstances pour lesquelles un crime pouvait être pardonné par le souverain. ${ }^{27}$ En effaçant tout, Henri IV ne laissait-il pas sous-entendre que l'idéal chevaleresque ne serait plus considéré en France?

Héritier d'une monarchie affaiblie, d'une situation politique plus que confuse, Henri IV tenta de sortir la France du marasme en liant la fidélité de ses sujets à sa personne. Mais, après 30 ans de guerres civiles, les intérêts étaient si divers, les appétits si aiguisés que le roi ne put satisfaire tout le monde. Quelques fidèles de la première heure se sentirent parfois oubliés par la manne royale alors que leurs anciens ennemis profitaient des largesses du roi; ils firent savoir à l'occasion leur mécontentement. Toutefois, le charisme personnel d'Henri IV réussit à lui garantir un règne somme toute assez calme. 
Les événements qui suivront son assassinat, la cabale des Grands contre la régente Marie de Médicis qui pourtant tentait de suivre les politiques établies par le roi défunt, qui essayait à coup d'argent de maintenir leur soutien à la couronne, montrent bien que la garantie de la paix socio-politique dépendait de la fidélité au roi-individu plutôt que de la fidélité à un royaume qui, pour beaucoup, demeurait encore quelque chose d'abstrait.

La voie empruntée par Henri IV pour gagner la fidélité de ses sujets nous éclaire donc sur la perception qu'avaient alors les Français des institutions du royaume. Le succès du roi reposa sur le contact personnel qu'il put établir avec la population. En pacifiant les villes et les régions une à une, en signant des traités avec des individus avant de proclamer en 1598 un édit s'appliquant à l'ensemble du royaume, le premier Bourbon ne nous indique-t-il pas que ses sujets voulaient d'abord s'attacher à un individu - le roi - plutôt qu'à une institution - la monarchie - ou à une nation - la France?

Malgré les réserves que certains de ses partisans ont pu manifester à l'égard de la clémence d'Henri IV, la bienveillance de ce dernier deviendra partie intégrante de sa légende. Le pardon royal prendra sous le règne des Bourbon une signification spéciale. Avant le couronnement de Louis XIII en 1610, les rois de France n'avaient pas coutume de libérer des prisonniers pour célébrer leur montée sur le trône. ${ }^{28}$ Non seulement Louis XIII élargira-t-il des détenus mais des centaines d'oiseaux seront relachés dans le ciel de Reims lors de la cérémonie du sacre. Grâce à Henri IV, la clémence deviendra un symbole incontesté de la puissance souveraine en France.

\section{Université McGill}

\section{Notes}

1. B[ibliothèque] N[ationale], Ms. fr. 18417, fol. 184-184v.

2. Seules sont exemptes de ce pardon les personnes associées à l'assassinat d'Henri III perpétré le ler août 1589. A[rchives] N[ationales], X1a 9230, fol. 122.

3. Discours au vrai de ce qui s'est passé en l'armée conduite par sa majesté, depuis son avènement à la Couronne, jusqu'à la prise des faubourgs de Paris. Et de là jusques à la prise de la ville d'Alençon, (s. 1., 1589), p. 5.

4. Si le droit médiéval accordait à plusieurs autorités la faculté de grâcier dans le cadre de leur justice, le XVIe siècle verra le pardon devenir prérogative royale. L. Le Roy, Les Politiques d'Aristote (Paris, 1568), p. 508; J. Bodin, Les six livres de la République (Paris, 1583), pp. 236-238; L. Charondas Le Caron, Pandectes ou digestes du droit français (Paris, 1637 [1596]\}, pp. 64-67; P. Poisson de la Bodinière, Traité de la majesté royale en France (Paris, 1597), pp. 32-33; B. De La Roche Flavin, Treize livres des parlements de France (Bordeaux, 1617), p. 9. Sur l'évolution du droit de grâce en France, voir: J. Foviaux, La rémission des peines et des condamnations. Droit monarchique et droit moderne (Paris, 1970). 
5. A. Arnauld, Coppie de l'Anti-Espagnol fait à Paris (s. 1., 1590), p. 49. Panégyrique au Très-Chrétién Henri IV (s. 1., 1590), p. 99v-100.

6. B. N., Ms. fr. 15900 , fol. 440 .

7. N. Davis, Fiction in the Archives. Pardon Tales and their Tellers in Sixteenth Century France (Stanford, 1987); J. G. Murphy, "Forgiveness and Resentment", dans J. G. Murphy et J. Hampton, Forgiveness and Mercy (Cambridge, 1988), pp. 14-34.

8. Sur cet aspect de la clémence, voir: D. Ménager, "La politique du don dans les derniers chapitres de Gargantua," dans The Journal of Medieval and Renaissance Studies, Vol. 8, No. 2(1978) pp. 179-191. Pour un exemple concret, voir les "Lettres patentes portant rémission à Jacques de Maillé d'avoir suivi la Ligue," données au Mans en décembre 1589, A. N., X1a 8640, fol. 99. Maillé, en échange du pardon royal, promet "de nous être si fidèle à l'avenir que nous aurons toute occasion de nous contenter de lui." Ces lettres sont enregistrées par le parlement de Tours le 10 janvier 1590: A. N., Xla 9230, fol. $154 \mathrm{v}$.

9. Des articles relatifs aux impositions qui se lèvent sur les rivières Rhône, Saône et Loire se retrouvent ainsi à l'article VIII de l'Edit et déclaration du roi, sur la réduction de la ville de Lyon, sous son obéissance (Lyon, 1594). Des mesures visant à prévenir les débordements de la Loire sont prévues dans l'Edit du roi sur la réduction de la Ville d'Orléans en son obéissance, (Orléans, 1594), article IX.

10. M. Hurault, Discours sur l'état de la France (Chartres, 1591), pp. 83-84.

11. Il "faut distribuer les loyers selon la vertu, et les peines selon les délits." L. Le Roy, Les politiques d'Aristote, p. 591. De l'obéissance dîe au Prince. Pour faire cesser les armes et rétablir la Paix en ce royaume (Caen, 1590), pp. 25-26. Panegyric au Très-Chrétien Henri IIII. Roi de France et de Navarre, par le S. D. I. E. S. L. (Tours, 1590), p. 50. Sur ce sujet, voir: P.-L. Vaillancourt, "Tolérance et clémence dans quelques traités politiques à l'automne de la Renaissance," dans La Tolérance: naissance et affirmation d'une idée de l'époque moderne (Poitiers, 1989), pp. 117-130.

12. Satyre Ménipée du Catholicon d'Espagne, et de la tenue des États de Paris (s. 1., 1593), p. 226-228.

13. Fidèle avertissement du seigneur Vasco Figueiro, gentilhomme Portugais, aux rebelles Français, de se retirer de la faction de Philippe II roi d'Espagne, de peur qu'ils ne tombent sous sa tyrannique domination. Et de retourner à l'obéissance de leur roi naturel et légitime (s. 1., 1591), pp. 59-60. A. Arnauld, La fleur de Lys (s. 1., s. d.), pp. 18-22.

14. La Satyre Ménipée se termine sur deux petits poèmes consacrés à ce thème (pp. 254-255). On trouve les vers suivants dans l'un d'entre eux:

C'est bien une vertu belle entre les plus belles,

D'être doux aux vaincus, et pardonner à tous:

Mais gardez-vous du trop, même envers les rebelles,

Car César en mourut grand Prince comme vous.

Les partisans d'Henri IV craignaient comme la peste la mort du roi, que celle-ci survienne lors d'une bataille ou par la main d'un assassin. Sur ce sujet voir: M. De Waele, "Pour la sauvegarde du roi et du royaume: l'expulsion des Jésuites de France à la fin des guerres de religion," à paraître dans Canadian Journal of History/Annales Canadiennes d'Histoire, Vol. 29, No.2. 
15. A ce sujet, signalons que dans la Bible le pardon est toujours conditionnel au repentir: "Forgive", dans A Theological Word Book of the Bible, ed. Alan Richardson (New York, 1951), pp. 85-86. La plupart des auteurs français du XVI ${ }^{\mathrm{e}}$ siècle affirment que le retour vers Dieu d'une personne déchue commençe par la prise de conscience de son péché: M. Wolfe, The conversion of Henri IV. Politics, Power, and Religious Belief in Early Modern France (Cambridge, 1993), pp. 9-16.

16. "J'ai avis comme assez bon qu'il se dresse une nouvelle Ligue. A laquelle on a convié par écrit un gentilhomme de notre quartier bon capitaine qui promet de mettre les lettres es mains du roi." B. N., Dupuy 819, fol. 105.

17. "Lettre de G. Du Vair à J. A. de Thou", 16 juillet 1599, B. N., Dupuy 802, fol. 40.

18. D. Drouin, Le miroir des rebelles traitant de l'excellence de la Majesté Royale, et la punition de ceux qui se sont élevés contre icelle (Tours, 1592), p. 264.

19. D. Drouin, Le miroir des rebelles, p. 192-215. Ce discours se retrouvait déja dans le Panégyrique au Très-Chrétien Henri IV, pp. 125-135.

20. L'allouette, Des affaires d'État. Des finances, du prince et de sa noblesse (Metz, 1597), p. 95; M. Hurault, Excellent et libre discours sur l'état présent de la France (s. 1., 1588), pp. 35-36.

21. M. Hurault, Discours sur l'état de la France, pp. 25-26; Panegyric au Très-Chrétien Henri III, pp. 65-68.

22. Le panégyrique adressé au roi de la part de ses bons sujets de sa ville de Paris (s. 1., 1590), p. 6; G. Joly, Panégyrique au roi Henri IIII, p. 9.

23. "La principale marque de souveraineté est la droite et souveraine administration de la justice", L. Charondas Le Caron, Pandectes, p. 2.

24. F. de Clary, Remontrance faite au Grand Conseil du roi sur le rétablissement requis par les officiers du roi qui ont suivi la Ligue (Caen, 1591). Cet argument avait déjà été présenté dans le Panegyric au Très-Chrétien Henri IV, p. 119. Demeuré à Paris sur ordre d'Henri III, François de Clary avait dû demander à Henri IV des lettres patentes lui permettant de réintégrer sa charge au grand conseil. Ces lettres datées du 21 novembre 1589 seront enregistrées le 12 décembre suivant: A. N., V5 1227, fol. 7.

25. G. Joly, Panégyrique au roi Henri IIII, p. 68. P. Poisson de la Bodinière, Traité de la majesté royale en France, pp. 32-33.

26. N. Davis, Fiction in the Archives, pp. 48-57.

27. J. Papon, Trias judiciel du Second Notaire (Lyon, 1575), p. 466-471, cité dans: N. Davis, Fiction in the Archives, p. 12. Voir également: A. Soman, "Les procès de sorcellerie au parlement de Paris (1565-1640)," dans Annales E.S.C., Vol. 32, No. 4, 1977, p. 790-814.

28. R. A. Jackson, Vive le Roi! A History of the French Coronation from Charles $V$ to Charles X (Chapel Hill, 1984), pp. 94-98. 